\title{
Some Applications of Bayes' Rule in Probability Theory to Electrocatalytic Reaction Engineering
}

\author{
Thomas Z. Fahidy \\ Chemical Engineering Department, University of Waterloo, 200 University Avenue West, Waterloo, ON, Canada N2L 3G1 \\ Correspondence should be addressed to Thomas Z. Fahidy, tfahidy@engmail.uwaterloo.ca \\ Received 7 February 2011; Accepted 25 April 2011 \\ Academic Editor: Carlos F. Zinola \\ Copyright () 2011 Thomas Z. Fahidy. This is an open access article distributed under the Creative Commons Attribution License, \\ which permits unrestricted use, distribution, and reproduction in any medium, provided the original work is properly cited. \\ Bayesian methods stem from the principle of linking prior probability and conditional probability (likelihood) to posterior \\ probability via Bayes' rule. The posterior probability is an updated (improved) version of the prior probability of an event, through \\ the likelihood of finding empirical evidence if the underlying assumptions (hypothesis) are valid. In the absence of a frequency \\ distribution for the prior probability, Bayesian methods have been found more satisfactory than distribution-based techniques. \\ The paper illustrates the utility of Bayes' rule in the analysis of electrocatalytic reactor performance by means of four numerical \\ examples involving a catalytic oxygen cathode, hydrogen evolution on a synthetic metal, the reliability of a device testing the quality \\ of an electrocatalyst, and the range of Tafel slopes exhibited by an electrocatalyst.
}

\section{Introduction}

In a comprehensive overall treatment of the subject matter, Bockris and Khan [1] discuss electrocatalysis with respect to various physicochemical properties of substance and surface, for example, exchange current density, work function, bond strength, metal complexes, trace elements adatom effects, enzymatic catalysis, poisons, crystal face effects, and so forth, under the aegis of "phenomenological electrode kinetics". In the domain of ERE, the assessment of electrocatalyst (EC) performance also includes additional parameters related to catalyst preparation (i.e., possible defectiveness in specimens), cell construction, and human factors affecting reactor output.

This paper was written with this dichotomy in mind, from the vantage point of the electrochemical engineer, whose responsibilities dealing with production quota, the possibility of (temporary) reactor breakdown, safety, and environmental considerations reach well beyond purely scientific quantities. Major tools for dealing with these responsibilities are provided by probability-based (e.g., statistical) methods. Bayes' rule is one such tool, whose specific application to scenarios with EC is the subject of this article.

\section{Brief Theory}

Following a concise definition [2] for the purposes of this paper, Bayes' rule for two events may be expressed as

$$
P(A / B)=\frac{P(B / A) P(A)}{P(B)},
$$

where $P(A / B)$ is the probability of event $A$ occurring if event $B$ has already occurred, and

$$
P(B)=P(B / A) P(A)+P\left(B / A^{\prime}\right) P\left(A^{\prime}\right),
$$

which is the probability of event $B$ occurring, given the conditional probabilities (likelihoods) $P(B / A)$ relating it to event $A$ and $P\left(B^{\prime} / A\right)$ relating it to its opposite event $A^{\prime}$. In an EC reactor a case for Bayes' rule would exist, for instance, when the loss of effectiveness in an EC may or may not be due to premature detachment of the catalytic layer. If $A$ is the event of detachment and $B$ is the event of deterioration (demise) of the EC, then $B / A$ would be the event of demise due to layer detachment, $A^{\prime}$ the event of a nondetachment cause of deterioration, and $B / A^{\prime}$ the event of deterioration due to a nondetachment cause. In terms of event probabilities, (1) yields the probability $P(A / B)$ that 
TABLE 1: Postulated distribution pattern of 137 failure occurrences over a fixed time period in three independently operating (hypothetical) electrolytic plants using identical catalytic oxygen cathodes (Application 1).

\begin{tabular}{|c|c|c|c|}
\hline \multirow{2}{*}{ Source of failure } & \multicolumn{3}{|c|}{ Number of cathode failures over a fixed period of operation } \\
\hline & Plant $1\left(B_{1}\right)$ & Plant $2\left(B_{2}\right)$ & Plant $3\left(B_{3}\right)$ \\
\hline$A_{1}:$ Catalyst surface area ${ }^{(1)}$ & 12 & 14 & 10 \\
\hline$A_{2}:$ Pore volume ${ }^{(2)}$ & 7 & 8 & 9 \\
\hline$A_{3}:$ Binder content ${ }^{(3)}$ & 9 & 5 & 7 \\
\hline$A_{4}:$ Catalyst content ${ }^{(4)}$ & 6 & 4 & 5 \\
\hline$A_{5}:$ Human error ${ }^{(5)}$ & 14 & 11 & 16 \\
\hline Total number of failures & 48 & 42 & 47 \\
\hline \multicolumn{4}{|c|}{$\begin{array}{l}\text { (1) Electrolytic carbon type P33: specific surface area is less than stipulated } 1000 \mathrm{~m}^{2} / \mathrm{g} \text {. } \\
\text { (2) Electrolytic carbon type P33: specific pore volume is less than stipulated } 2.3 \mathrm{~cm}^{3} / \mathrm{g} \text {. } \\
\text { (3) PTFE binder content in electrode layer is less than stipulated } 10 \% \text {. } \\
\text { (4) CoTAA (dibenzotetraazaannulen cobalt II) catalyst content on carbon is less than stipulated } 15 \% \text {. } \\
\text { (5) Careless stack assemblage and general operation. }\end{array}$} \\
\hline
\end{tabular}

deterioration would occur as a result of layer detachment and not due to a different cause, for example, the decomposition of a binder, or the splitting of the electrode frame, and so forth. If $A_{1}, A_{2}, \ldots, A_{n}$ are mutually exclusive and exhaustive events, (1) and (2) are generalized to

$$
P\left(A_{k} / B\right)=\frac{P\left(B / A_{k}\right) P\left(A_{k}\right)}{\sum_{k=1}^{n} P\left(B / A_{k}\right) P\left(A_{k}\right)},
$$

taking into account all possible causes of deterioration (the denominator of (3) is also known as the total probability theorem [3]). A lucid discussion of the merits of Bayesian methods by Bulmer [4] and a short set-theoretic proof by Arnold [5] are amply complemented by a sizeable literature on probability and statistics dealing with the subject matter.

Specific exploratory applications to electrochemical processes and technology at various levels of complexity are relatively recent [6-10]. The paper illustrates, via four independent examples, the (potential) utility of Bayes' rule in ERE. Due to the currently insufficient availability of appropriate experimental information in the research literature, hypothetical numerical data are employed with the sole purpose of indicating the course of analysis to which appropriate experimental data could be subjected. With the intention of stimulating at least a modest appetite at present for Bayes' rule, the illustrations are realistic but uncomplicated on purpose.

\section{Illustration of the Utility of Bayes' Rule for ERE}

3.1. Application No. 1: Estimating the Most Likely Location of Oxygen-Cathode Failure. Table 1 contains the failurefrequency map of identical oxygen cathodes, assumed to possess the structure described by Wiesener and Ohms [11]. These mutually independent failures are stipulated to have occurred in three independently operating electrochemical plants. Denoting $A_{1}, A_{2}, \ldots A_{5}$ as the source-of-failure events and $B_{1}, B_{2}, B_{3}$ as the plant location events, the probability of failure arising, for example, from human error is given by

$$
\begin{aligned}
P\left(A_{5}\right) & =\sum_{k=1}^{3} P\left(A_{5} / B_{k}\right) P\left(B_{k}\right)=\frac{14}{48} \frac{48}{137}+\frac{11}{42} \frac{42}{137}+\frac{16}{47} \frac{47}{137} \\
& =\frac{41}{137}=0.2993
\end{aligned}
$$

which is about $30 \%$, and Bayes' rule:

$$
P\left(B_{j} / A_{5}\right)=\frac{P\left(A_{5} / B_{j}\right) P\left(B_{j}\right)}{P\left(A_{5}\right)}, \quad j=1,2,3,
$$

yields the probability of failure in any one of the three plants caused by human error: $P\left(B_{1} / A_{5}\right)=14 / 41=0.3415$; $P\left(B_{2} / A_{5}\right)=11 / 41=0.2683 ; P\left(B_{3} / A_{5}\right)=16 / 41=0.3802$. Thus, (next time) failure due to human error can be expected to be the least likely in Plant 2 and the most likely in Plant 3, although not significantly so with respect to Plant 1. The entire set $P\left(B_{k} / A_{j}\right), j=1,2,3, k=1,2, \ldots, 5$ of likelihoods, obtained in a manner similar to (5) is shown in Table 2 . The relatively largest failure probability, about $43 \%$, can be expected in Plant 1 on account of insufficient PTFE binder content in the electrode layer. The contents of Table 2 would guide plant operators in attempting to eliminate (or at least to reduce the extent of) the most likely cause that can be expected in each plant. They would also indicate what cautionary measures would be advisable in the design of future plants.

3.2. Application No. 2: The Effect of Prior Probability on the Anticipated Viability of an EC-Generated $\mathrm{H}_{2}$ Evolution Process. A recently developed electrocatalyst for a hydrogen evolution process, made up of certain synthetic metals, is expected to possess an exchange current $i_{0} \approx 100 \mu \mathrm{A} / \mathrm{cm}^{2}$ at design operating conditions in a pilot scale electrolyzer. Inspection of Trasatti's [12] "volcano plot" [13, 14] suggests that its catalytic property would presumably fall between that of iridium and gold. It is further anticipated that the novel 
TABLE 2: The complete set of probabilities computed via (5) in Application 1.

\begin{tabular}{lccc}
\hline Source-of-failure events & \multicolumn{3}{c}{$P\left(B_{j} / A_{k}\right)$} \\
& $B_{1}$ & $B_{2}$ & $B_{3}$ \\
\hline$A_{1}$ & 0.3333 & 0.3889 & 0.2778 \\
$A_{2}$ & 0.2917 & 0.3333 & 0.3750 \\
$A_{3}$ & 0.4286 & 0.2381 & 0.3333 \\
$A_{4}$ & 0.4000 & 0.2667 & 0.3333 \\
$A_{5}$ & 0.3415 & 0.2683 & 0.3902 \\
\hline
\end{tabular}

TABLE 3: The effect of prior probability $P(B)$ on decision possibilities related to a new CE (Application 2).

\begin{tabular}{lcc}
\hline$P(B) \%$ & $P(B / A) \%$ & $P\left(B / A^{\prime}\right) \%$ \\
\hline 20 & 67.4 & 2.5 \\
40 & 84.6 & 6.3 \\
60 & 92.5 & 13.2 \\
70 & 95.1 & 19.1 \\
80 & 97.1 & 28.8 \\
90 & 98.7 & 47.6 \\
\hline
\end{tabular}

$P(B / A)$ : the probability that a CE will be deemed acceptable upon the $Q_{2}$ test, if the results of the $Q_{1}$ test were positive.

$P\left(B / A^{\prime}\right)$ : the probability that a $C E$ will be deemed acceptable upon the $Q_{1}$ test, even if the results of the $Q_{1}$ test were negative.

catalyst would be less expensive than $\mathrm{Ir}$ and $\mathrm{Au}$, it would exhibit good dimensional/geometric stability as well as resistance to parasitic reactions due to possible contamination, and resistance to possible nonuniformity in current distribution. The design team postulates that if, on a pilotplant scale, electrode specimens will show no loss in catalytic activity up to the passage of $Q_{1} \approx 600 \mathrm{kAh} / \mathrm{dm}^{2}$ electric charge per unit area, then there should exist an a priori chance that a catalyst-carrying electrode (CCE), selected randomly from a lot of identically prepared specimens, can sustain its catalytic activity, at an acceptable level, up to the passage of $Q_{2} \approx 1200 \mathrm{kAh} / \mathrm{dm}^{2}$. During the $Q_{1}$ tests, $91 \%$ of the electrodes were found to be acceptable, but $89 \%$ of electrodes, which later failed the $Q_{2}$ tests, did not perform in a satisfactory manner. The design team (i) would proceed to consider commercial-scale implementation if there is at least a 95\% chance that a survivor of the $Q_{1}$-test would keep its catalytic activity up to the passage of $Q_{2}$, (ii) would abandon further research if favourable results were obtained in only one-fifth or less of the $Q_{1}$ tests.

The set of events of interest here, involving a randomly selected CCE, is defined as follows:

$A$ : results obtained during the passage of $Q_{1}$ are positive,

$A^{\prime}$ : results obtained during the passage of $Q_{1}$ are negative,

$B$ : the CCE is acceptable,

$B^{\prime}$ : the CCE is unacceptable,

$A / B: Q_{1}$-results were positive for an acceptable CCE, $A / B^{\prime}: Q_{1}$-results were positive for an unacceptable CCE,

$A^{\prime} / B: Q_{1}$-results were negative for an acceptable CCE,

$A^{\prime} / B^{\prime}: Q_{1}$-results were negative for an unacceptable CCE,

$B / A$ : a CCE which showed positive $Q_{1}$-test results is found acceptable,
$B / A^{\prime}$ : a CCE which showed negative $Q_{1}$-test results is found acceptable.

Consequently, the stipulations can be expressed in terms of their probabilities as follows: $P(A / B)=0.91 ; P\left(A^{\prime} / B^{\prime}\right)=$ $0.89 ; P\left(A / B^{\prime}\right)=1--P\left(A^{\prime} / B^{\prime}\right)=0.11$. Bayes' rule yields, therefore,

$$
\begin{aligned}
P(B / A) & =\frac{0.91 P(B)}{0.91 P(B)+0.11[1-P(B)]}, \\
P\left(B / A^{\prime}\right) & =\frac{0.09 P(B)}{0.09 P(B)+0.89[1-P(B)]} .
\end{aligned}
$$

Here, $P(B)$ is the prior probability of a CCE being acceptable. Its value, if not known experimentally, would be a matter of the designers' judgment. Table 3 indicates that in order to satisfy the $P(B / A) \geq 95 \%$ and $P\left(B / A^{\prime}\right) \geq 20 \%$ decision criteria simultaneously, the prior probability of a CCE passing the $Q_{2}$-test would have to be somewhat higher than $70 \%$. If the abandonment criterion were raised to a stricter $P\left(B / A^{\prime}\right) \geq 25 \%$ probability, $P(B)$ would have to be at least $77 \%$ for satisfying the two continuance conditions. Such results provide the design team with important knowledge for establishing proper testing protocols.

3.3. Application 3: Probing Claims Regarding the Reliability of a Catalyst Tester. A device for testing defects in a certain electrocatalyst (EC) is envisaged to be advertised by the catalyst producer, claiming that it is $97 \%$ reliable if the EC is defective, and $99 \%$ reliable when it is flawless. Independently from any testing device and based upon earlier experience, $4 \%$ of said EC may be expected to be defective upon delivery. In order to ascertain the true reliability of the device, Bayes' rule is applied to basic event set $A$ : the EC is defective; $A^{\prime}$ : the $\mathrm{EC}$ is flawless; $B$ : the EC is tested to be defective; $B^{\prime}$ : the EC is tested to be flawless, equipped with the full set of conditional events of interest here with their probabilities:

$B / A$ : EC is (known to be) defective, and tested defective, $P(B / A)=0.97$,

$B^{\prime} / A$ : EC is (known to be) defective, but tested flawless, $P\left(B^{\prime} / A\right)=1--P(B / A)=0.03$,

$B / A^{\prime}$ : EC is (known to be) defective, but tested defective, $P\left(B / A^{\prime}\right)=1--P\left(B^{\prime} / A^{\prime}\right)=0.01$,

$B^{\prime} / A$ : EC is (known to be) flawless, and tested flawless, $P\left(B^{\prime} / A^{\prime}\right)=0.99$.

The probabilities of events to be computed via Bayes' rule, shown in Table 4, point to the (vexingly) high possibility of rejecting flawless EC's (about 20\%) and the (vexingly) low possibility of identifying defective EC's (about 80\%) when the tester indicates defectiveness. These findings, hidden by the advertisement without Bayes' rule, should discourage its adoption for routine use.

3.4. Application 4: Probing Claims Regarding Tafel Slopes in an Electrocatalytic Oxidation of Methanol Process Envisaged for Fuel Cells. This example is motivated by an experimental study of Pt:Mo dispersed catalysts (PMDCs) for the electrooxidation of methanol in acid medium [15], assuming that a 
TABLE 4: Probabilities of flawlessness/defectiveness expected from an EC tester (Application No. 3).

\begin{tabular}{lrr}
\hline Event & Bayes' rule & Event probability \\
\hline EC tested defective, but found flawless & $P\left(A^{\prime} / B\right)=\frac{P\left(B / A^{\prime}\right) P\left(A^{\prime}\right)}{P\left(B / A^{\prime}\right) P\left(A^{\prime}\right)+P(B / A) P(A)}$ & 0.1983 \\
EC tested flawless, and found flawless & $P\left(A^{\prime} / B^{\prime}\right)=\frac{P\left(B^{\prime} / A^{\prime}\right) P\left(A^{\prime}\right)}{P\left(B^{\prime} / A^{\prime}\right) P\left(A^{\prime}\right)+P\left(B^{\prime} / A\right) P(A)}$ & 0.9987 \\
EC tested flawless, but found defective & $P\left(A / B^{\prime}\right)=\frac{P\left(B^{\prime} / A\right) P(A)}{P\left(B^{\prime} / A\right) P(A)+P\left(B^{\prime} / A^{\prime}\right) P\left(A^{\prime}\right)}$ \\
EC tested defective, and found defective & $P(A / B)=\frac{P(B / A) P(A)}{P(B / A) P(A)+P\left(B / A^{\prime}\right) P\left(A^{\prime}\right)}$ & 0.0013 \\
\hline
\end{tabular}

$P\left(A / B^{\prime}\right)+P\left(A^{\prime} / B^{\prime}\right)=P(A / B)+P\left(A^{\prime} / B\right)=1 ; P(A)=0.04 ; P\left(A^{\prime}\right)=1-0.04=0.96$.

different research team claims in a new experimental catalyst development program a $65-70 \mathrm{mV} / \mathrm{dec}$ Tafel slope range at low current densities, and a 255-265 $\mathrm{mV} / \mathrm{dec}$ at high current densities (in contrast with the 30-35 and $230-250 \mathrm{mV} / \mathrm{dec}$ ranges, resp. in the cited study). The polarization method chosen for investigating the claim is assumed to be $89 \%$ reliable when the claim cannot be verified and 99.5\% reliable when the claim can be verified. Defining events $A$ : the PMDC exhibits Tafel slopes below the claimed ranges and $B$ : the PMDC is found to exhibit Tafel slopes below the claimed ranges, the complementary events $A^{\prime}$ : the PMDC exhibits Tafel slopes within the claimed ranges and $B^{\prime}$ : the PMDC is found to exhibit Tafel slopes within the claimed ranges establish the basis for applying Bayes' theorem. Following the pattern shown by the previous applications, the conditional probabilities are $P\left(B^{\prime} / A\right)=0.11, P\left(B / A^{\prime}\right)=0.005, P(B / A)=$ 0.89 and $P\left(B^{\prime} / A^{\prime}\right)=0.995$.

The research team is assumed to report that $96 \%$ of the new PMDC possess the claimed Tafel slope ranges; Bayes' theorem yields $P\left(A^{\prime} / B\right)=0.1188 ; P\left(A / B^{\prime}\right)=0.0046$; $P\left(A^{\prime} / B^{\prime}\right)=0.9954 ; P(A / B)=0.8812$. The about $12 \%$ probability that a new catalyst complies with the claim although the polarization experiment indicates otherwise raises at least a reasonable doubt about the claim or the reliability of the experimental procedure, in spite of the satisfactory $P\left(A / B^{\prime}\right)$ and $P\left(A^{\prime} / B^{\prime}\right)$ values.

\section{Discussion and Final Remarks}

Perhaps the most striking feature of Bayes' rule is the amount of information that can be gleaned from a few uncomplicated probability ratios (the fact that Bayesian methods are at present more than two hundred years old is equally impressive). Within the Bayesian framework, a prior event probability is updated to a posterior probability of that event by means of a likelihood. The latter provides the (conditional) probability of corroborating the a-priori stated hypothesis; this aspect is numerically illustrated in the Appendix.

The examples presented in this paper provide a small "window" to the realm of Bayesian methods whose further exploration in electrochemical science and engineering requires further work. Bayes' rule is just one of many other mathematical devices of applied probability theory with potential interest to the field.

\section{Appendix}

\section{Bayes' Rule: Short Analysis and Illustration via Application No. 3}

Let $\mathrm{AB}$ and $\mathrm{BA}$ denote the combined event of both events $\mathrm{A}$ and $\mathrm{B}$ occurring, the order of occurrence being immaterial. The veracity of the statement $P(A B)=P(B A)=$ $P(B / A) P(A)=P(A / B) P(B)$ and of (1) immediately follows. Accounting for complementary events $A^{\prime}$ and $B^{\prime}$ the (total) probabilities $P(A)=P(A / B) P(B)+P\left(A / B^{\prime}\right) P\left(B^{\prime}\right)$ and $P(B)=P(B / A) P(A)+P\left(B / A^{\prime}\right) P\left(A^{\prime}\right)$ are mirror images of each other. In Application No. $3 P(B / A) P(A)=$ $(0.97)(0.04)=0.0388$ is the posterior probability of an EC being defective vis-à-vis prior probability. $P(A)=$ 0.04. Similarly, $P\left(B / A^{\prime}\right) P\left(A^{\prime}\right)=(0.01)(0.96)=0.0096$ is the posterior probability of EC-defectiveness vis-à-vis prior complementary probability $P\left(A^{\prime}\right)=0.96$; the total probability of event $B$ is $0.04+0.0096=0.0484$. Bayes' rule yields $P(A / B)=0.0388 / 0.0484=0.8017$, that is the posterior probability that an EC tested defective is, indeed, defective. It is appreciably less than the claimed (i.e. prior) probability of 0.97 .

\section{Acknowledgments}

Utilities provided by the University of Waterloo and the Natural Sciences and Engineering Research Council of Canada (NSERC) are gratefully acknowledged.

\section{References}

[1] J. O'M. Bockris and S. U. M. Khan, Surface Electrochemistry, Plenum, New York, NY, USA, 1993.

[2] R. Porkess, Collins Internet-Linked Dictionary of Statistics, Harper Collins College, Glenview, Ill, USA, 2nd edition, 2005.

[3] E. B. Manoukian, Modern Concepts and Theorems of Mathematical Statistics, Springer, New York, NY, USA, 1986.

[4] M. G. Bulmer, Principles of Statistics, Dover, New York, NY, USA, 2nd edition, 1979.

[5] S. F. Arnold, Mathematical Statistics, Prentice Hall, Englewood Cliffs, NJ, USA, 1990.

[6] T. Z. Fahidy, "On the utility of certain decision theory techniques in electrochemical process design," in Current Topics in Electrochemistry, vol. 7, pp. 119-123, Research Trends, Trivandrum, India, 2000. 
[7] T. Z. Fahidy, "An application of Bayesian risk theory to electrochemical processes," Electrochimica Acta, vol. 49, no. 910, pp. 1397-1401, 2004.

[8] T. Z. Fahidy, "Bayesian updating of electrochemical process parameters via natural conjugate probability distributions," Electrochimica Acta, vol. 49, no. 27, pp. 5013-5021, 2004.

[9] T. Z. Fahidy, "Probabilstic methods in the analysis of certain electrochemical systems," in Recent Research Developments in Electrochemistry, S. G. Pandalai, Ed., vol. 6, pp. 116-117, Transworld Research Network, Trivandrum, India, 2003.

[10] T. Z. Fahidy, "Electrochemical applications of net-benefit analysis via Bayesian probabilities," Journal of Applied Electrochemistry, vol. 37, no. 6, pp. 747-752, 2007.

[11] K. Wiesener and D. Ohms, "Electrocatalysis for environmentally orientated electrochemical processes and environmental protection," in Environmental Oriented Electrochemistry, C. A. C. Sequeira, Ed., p. 694, Elsevier, Amsterdam, The Netherlands, 1994.

[12] S. Trasatti, "Work function, electronegativity, and electrochemical behaviour of metals. III. Electrolytic hydrogen evolution in acid solutions," Journal of Electroanalytical Chemistry, vol. 39, no. 1, pp. 163-184, 1972.

[13] J. O’M. Bockris and S. U. M. Khan, loc. cit., Section 3.14.3, Fig. 3.23 , p. 268

[14] J. O'M. Bockris and S. U. M. Khan, loc. cit., Section 3.21.6, Fig. 3.31, p. 293.

[15] A. Oliveira Neto, J. Perez, W. T. Napporn, E. A. Ticianelli, and E. R. Gonzalez, "Electrocatalytic oxidation of methanol: study with Pt:Mo dispersed catalysts," Journal of the Brazilian Chemical Society, vol. 11, no. 1, pp. 39-43, 2000. 


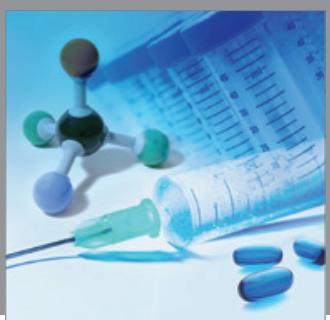

International Journal of

Medicinal Chemistry

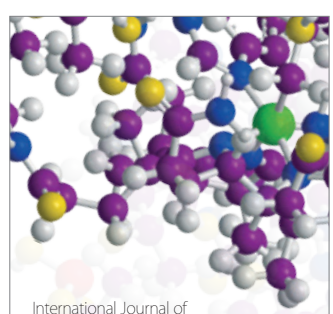

Carbohydrate Chemistry

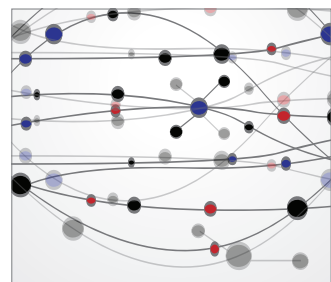

The Scientific World Journal
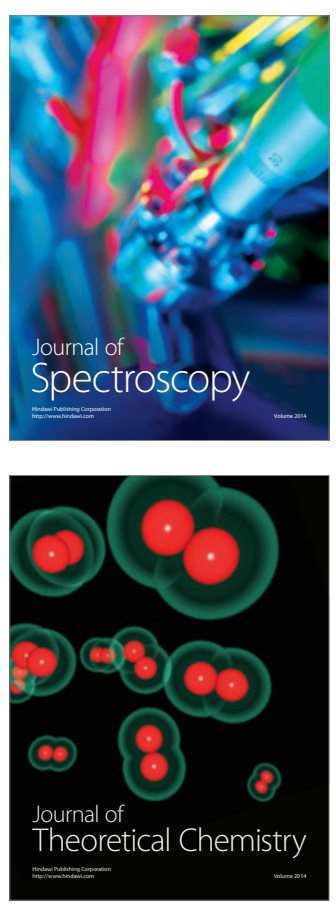
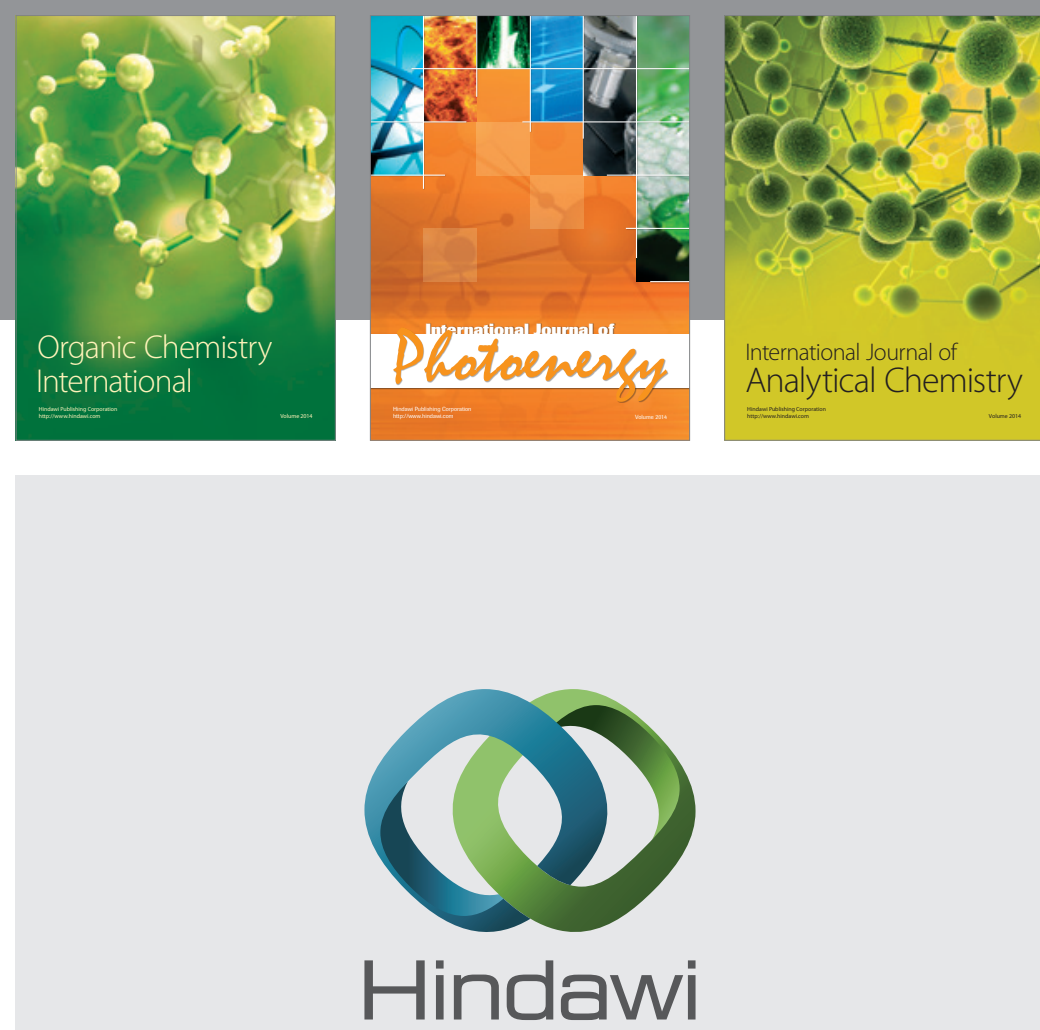

Submit your manuscripts at

http://www.hindawi.com
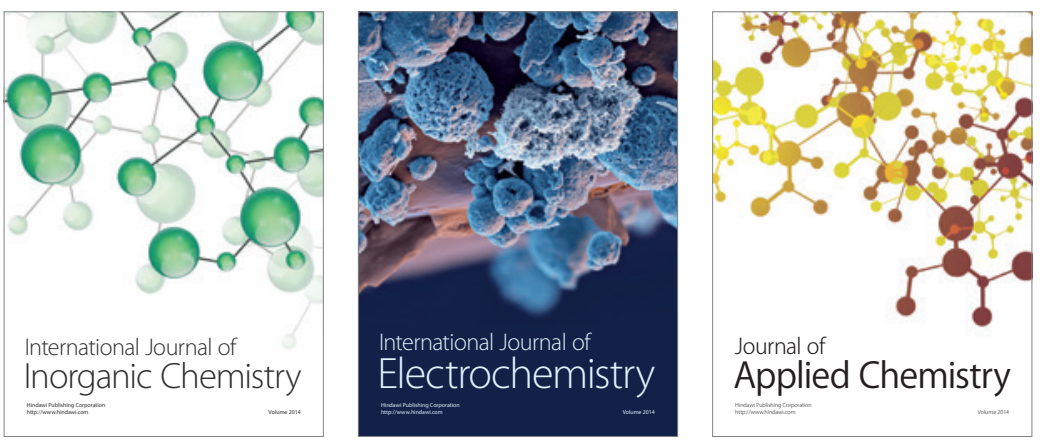

Journal of

Applied Chemistry
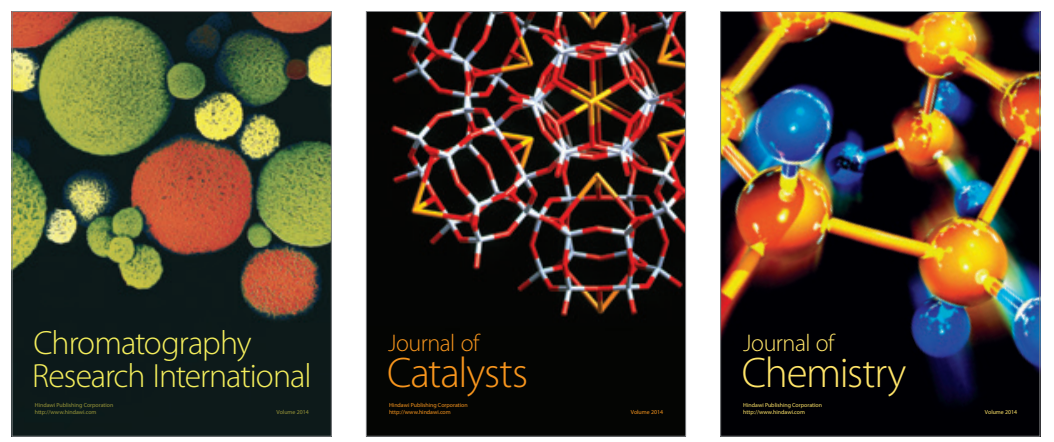
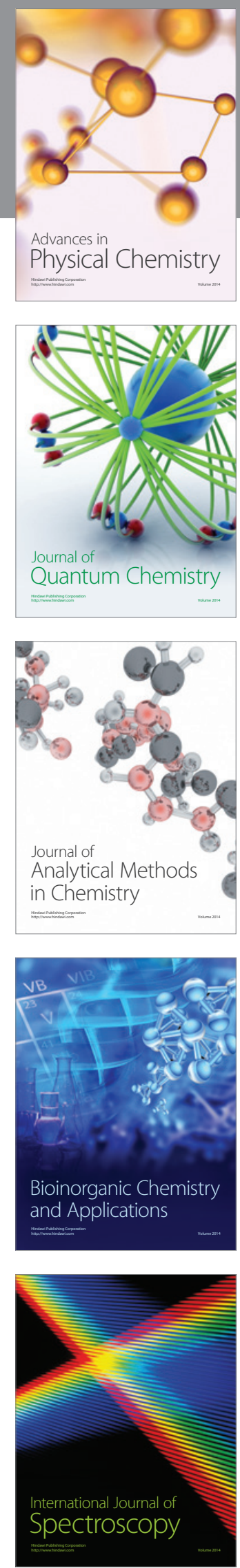